\title{
Statistical and Machine Learning Approaches to Predict Gene Regulatory Networks From Transcriptome Datasets
}

\author{
Keiichi Mochida ${ }^{1,2,3,4 *}$, Satoru Koda ${ }^{5}$, Komaki Inoue ${ }^{1}$ and Ryuei Nishii ${ }^{*}$ \\ ${ }^{1}$ Bioproductivity Informatics Research Team, RIKEN Center for Sustainable Resource Science, Yokohama, Japan, \\ ${ }^{2}$ Microalgae Production Control Technology Laboratory, RIKEN Baton Zone Program, RIKEN Cluster for Science, \\ Technology and Innovation Hub, Yokohama, Japan, ${ }^{3}$ Institute of Plant Science and Resources, Okayama University, \\ Kurashiki, Japan, ${ }^{4}$ Kihara Institute for Biological Research, Yokohama City University, Yokohama, Japan, ${ }^{5}$ Graduate School \\ of Mathematics, Kyushu University, Fukuoka, Japan, ${ }^{6}$ Institute of Mathematics for Industry, Kyushu University, Fukuoka, \\ Japan
}

\section{OPEN ACCESS}

Edited by:

Chuang Ma,

Northwest A\&F University, China

Reviewed by:

John Louis Van Hemert, DuPont Pioneer, United States

Luis Mendoza,

National Autonomous University

of Mexico, Mexico

${ }^{*}$ Correspondence:

Keiichi Mochida

keiichi.mochida@riken.jp

Ryuei Nishii

nishii@imi.kyushu-u.ac.jp

Specialty section:

This article was submitted to Technical Advances in Plant Science,

a section of the journal

Frontiers in Plant Science

Received: 25 August 2018 Accepted: 14 November 2018 Published: 29 November 2018

Citation:

Mochida K, Koda S, Inoue K and Nishii R (2018) Statistical and Machine Learning Approaches to Predict Gene Regulatory Networks

From Transcriptome Datasets.

Front. Plant Sci. 9:1770.

doi: 10.3389/fp/s.2018.01770
Statistical and machine learning (ML)-based methods have recently advanced in construction of gene regulatory network (GRNs) based on high-throughput biological datasets. GRNs underlie almost all cellular phenomena; hence, comprehensive GRN maps are essential tools to elucidate gene function, thereby facilitating the identification and prioritization of candidate genes for functional analysis. High-throughput gene expression datasets have yielded various statistical and ML-based algorithms to infer causal relationship between genes and decipher GRNs. This review summarizes the recent advancements in the computational inference of GRNs, based on large-scale transcriptome sequencing datasets of model plants and crops. We highlight strategies to select contextual genes for GRN inference, and statistical and ML-based methods for inferring GRNs based on transcriptome datasets from plants. Furthermore, we discuss the challenges and opportunities for the elucidation of GRNs based on largescale datasets obtained from emerging transcriptomic applications, such as from population-scale, single-cell level, and life-course transcriptome analyses.

\section{Keywords: machine learning, gene regulatory network, sparse modeling, transcriptome, time series analysis}

\section{INTRODUCTION}

Gene regulatory networks (GRNs) represent the causal relationship between genes regulating cellular functions (Barabasi and Oltvai, 2004; Blais and Dynlacht, 2005). GRNs play important roles in cellular regulatory systems such as signal transduction and transcriptional regulation, which underlie almost all cellular phenomena. Therefore, comprehensive GRN maps are essential tools to elucidate gene function, thereby facilitating interpretations of biological processes, such as cellular differentiation and response to environmental stimuli at system-level (Lopez-Maury et al., 2008), and enabling the identification and prioritization of candidates of genes for molecular regulators and biomarkers (van Dam et al., 2018).

A number of approaches have been proposed for reconstruction of GRNs based on highthroughput biological datasets. Transcriptome datasets, usually from time-series samples, have 
enabled us to infer gene expression networks using various statistical and machine learning ML-based algorithms (Dewey and Galas, 2010). The inferred GRNs are complementary to gene networks obtained from other types of data: transcription factor networks based on high-throughput methods to examine the interaction between transcription factors (TFs) and DNAbinding sites on target genes (Ikeuchi et al., 2018), and gene networks genetically determined using large-scale populations and mutant panels (Fuxman Bass et al., 2015; Hanson et al., 2018).

In this review, we provide an overview of recent advances in the computational inference of GRNs, based on large-scale transcriptome sequencing datasets of model plants and crops. We highlight statistical methods, including sparse modeling and machine-learning methods, for inferring GRNs based on transcriptomic datasets from plants. Furthermore, we discuss the challenges and opportunities for the elucidation of GRNs based on large-scale datasets obtained from emerging transcriptomic applications, based on population-scale, single-cell level, or lifecourse analyses.

\section{CONTEXTUAL GENE SELECTION}

Since statistical and ML-based approaches for GRN inference often have high computational complexities with highdimensional transcriptome datasets, selection of contextual genes may be a strategy to solve the NP-hard nature (large number of genes to limited number of data points). Differentially expressed genes, including those encoding transcription factors (TFs), across spatial and temporal transcriptome datasets, form a context filter widely used to select genes for GRN inference. To predict GRNs involved in stem cell regulation in Arabidopsis roots using spatial and temporal transcriptome data, de Luis Balaguer et al. (2017) selected 1,625 genes, identified by their differential expression in the stem cells, and focused on $201 \mathrm{TF}$ genes to infer GRNs, based on their observation of enriched GO categories, such as the regulation of transcription and TF activity in the genes. Comparing the DNA-binding capabilities between selected TFs and promoter regions of selected genes, such as DEGs and co-expressed genes, would also facilitate further narrowing down of genes that would be potentially regulated by the selected TFs in the TF network (Ni et al., 2016; Wilkins et al., 2016; Hickman et al., 2017). For example, (Wilkins et al., 2016) identified 5,447 putative target genes for 445 TFs by searching for known cis-regulatory motifs in open promoter regions, determined by an ATAC-seq analysis to select genes and TFs involved in a GRN that responds to environmental stimuli. Constructing an initial network by assumption-free methods, such as information theory-based methods or co-expression analysis, would be feasible to minimize false-positive edges with high computational efficiency in GRN inference, enabling us to apply statistical or ML-based methods to examine causalities between genes with respect to each local subnetwork in the initial networks (Liu et al., 2016). To predict the drought stress-responsive GRN in sunflower, Marchand et al. (2014) selected 145 genes that were co-expressed under drought stress conditions, and subsequently used a Gaussian graphical modeling method and a Random Forest method to infer the robust edges (Marchand et al., 2014). In addition to these approaches, genetics-based approaches to identify genotype-phenotype relationship can provide plausible sets of genes that are involved in a GRN. Calabrese et al. (2017) adopted an approach, integrating GWAS and co-expression network analysis, to narrow down the causal genes for bone mineral density, suggesting the feasibility of genetics-based selection of genes whose interplay underlies biological processes related to traits of interest. Through analysis of eQTL and eQTL-guided co-expression network, Basnet et al. (2016) identified candidate genes that genetically regulate the fatty acid composition in Brassica rapa seeds, based on cis- and trans-QTLs, detected by the eQTL analysis; this demonstrated that eQTLs can suggest a causal relationship between genes, complementary to networks inferred by computational methods.

\section{GRN INFERENCE APPROACHES}

\section{Statistics-Based Approaches}

Since time-series datasets contain dynamic information, which assists us in understanding temporal dynamics of various biological processes, statistics-based approaches have been often applied to time-series transcriptome datasets to infer GRNs (Table 1). The Autoregressive exogenous variables (ARX) model, a kind of state-space model, is useful to describe time-varying processes observed in time-series datasets, which enable us to reconstruct a GRN in combination with sparse estimation algorithms. Fused Lasso, a sparse estimation algorithm, was employed to reconstruct GRNs with time-series expression datasets from Escherichia coli, Mycobacterium tuberculosis, and Mus musculus (Omranian et al., 2016) ${ }^{1}$. In a model grass Brachypodium distachyon, Koda et al. (2017) had formulated gene-gene temporal interactions for 3,621 periodically expressed genes, observed in a time-series RNA-Seq dataset based on ARX models, combined with a statistical sparse estimation method Group SCAD (Smoothly Clipped Absolute Deviation), a kind of L_1 regularization technique to estimate sparse GRNs, and predicted GRNs containing 2,187 genes and 3,107 directed edges. The Inferelator algorithm ${ }^{2}$, a kind of sparse regression approach (Greenfield et al., 2013), was also applied to infer an environmental gene regulatory influence network (EGRIN) from datasets of time-series transcriptome (RNA-Seq) and chromatin accessibility (ATAC-seq) in five tropical Asian rice cultivars to understand their physiological response to high temperature and water deficiency under agricultural field conditions (Wilkins et al., 2016). de Luis Balaguer et al. (2017) developed GENIST ${ }^{3}$, based on a dynamic Bayesian network algorithm, and applied it to infer GRN from cell-type specific and time-series transcriptome data of Arabidopsis root stem cells. Comparing its performance in GRN inference with previously published methods, the authors

\footnotetext{
${ }^{1}$ https://github.com/omranian/inference-of-GRN-using-Fused-LASSO

${ }^{2}$ https://sites.google.com/a/nyu.edu/inferelator/home

${ }^{3} \mathrm{https} / / /$ github.com/madeluis/GENIST
} 
TABLE 1 | Examples of statistics-based and machine learning-based algorithms used for GRN inference in plants and other species.

\begin{tabular}{|c|c|c|c|}
\hline Data type & Algorithm & Organism & Reference \\
\hline \multicolumn{4}{|l|}{ Statistics-based algorithms } \\
\hline \multirow[t]{6}{*}{ Time series } & Fused LASSO regression & Escherichia coli & Omranian et al., 2016 \\
\hline & & Mycobacterium tuberculosis & \\
\hline & & Mus musculus & \\
\hline & ARX model and GroupSCAD & Brachypodium distachion & Koda et al., 2017 \\
\hline & Inferelator & Oryza sativa (five tropical Asian rice) & Wilkins et al., 2016 \\
\hline & GENIST & Arabidopsis thaliana & de Luis Balaguer et al., 2017 \\
\hline \multirow[t]{2}{*}{ Non-time series } & CLR & Zea maize & Xiong et al., 2017 \\
\hline & PRC & Saccharomyces cerevisiae & Blum et al., 2018 \\
\hline \multicolumn{4}{|l|}{ Machine learning-based algorithms } \\
\hline Various biological and experimental conditions & MinReg & Fusarium graminearum & Guo et al., 2016 \\
\hline Various tissues & GENIE3 & Zea maize & Huang et al., 2018 \\
\hline Time series (spatial and temporal) & DFG & Arabidopsis thaliana & Varala et al., 2018 \\
\hline
\end{tabular}

demonstrated that the GENIST algorithm outperformed with datasets used in DREAM 4 challenge 2.

There also exist examples of statistics-based approaches to infer GRNs using non-time-series transcriptome datasets (Table 1). Xiong et al. (2017) inferred GRNs to identify key genes in the maize seed development process with RNA-Seq data from various tissues: embryos, endosperms, whole seeds, and other tissues, by Context Likelihood of Relatedness (CLR $\left.{ }^{4}\right)$ (Faith et al., 2007), which is a mutual information (MI)-based GRN inference approach (Xiong et al., 2017). They inferred gene regulatory relationship based on z-score of MI between a TF gene and a non-TF or another TF gene, and generated a GRN composed of 10,932 nodes and 48,740 edges. They also verified eight regulatory relations between $\mathrm{TF}$ and non-TF genes, through yeast one-hybrid $(\mathrm{Y} 1 \mathrm{H})$ assay, to assess TF-promoter binding, and assessed the Opaque-2 TF network, inferred in the GRN, by comparing it with previously identified regulatory network based on the results from ChIP-seq analysis and RNASeq analysis of its mutants. Since GRNs inferred by MI-based approach basically show undirected graph, regulatory relations between genes are usually based on their putative function, i.e., TF-encoding genes or non-TF genes. To estimate regulatory relations between genes, Blum et al. (2018) developed a GRN inference algorithm based on partial response coefficients (PRC), and assessed its performance on synthetic datasets, as well as transcriptome datasets, from gene knockout mutants of yeast (Kemmeren et al., 2014), demonstrating its superior performance for GRN inference in studies with large-scale knockout mutant resources (Blum et al., 2018).

\section{Machine Learning-Based Approaches}

Machine learning, an area of computer science that offers data-driven prediction, has attracted wide attention for its various applications in modern biology (Camacho et al., 2018; Webb, 2018), besides putting forth its strength in GRN inference (Table 1). Guo et al. (2016) applied the MinReg

${ }^{4} \mathrm{http} / / / \mathrm{m} 3 \mathrm{~d} . \mathrm{mssm}$. edu/network_inference.html algorithm $^{5}$, based on a derivative of Bayesian networks, and a greedy algorithm, to infer the global GRN in Fusarium graminearum with 27 (9 experiments with three biological replicates) and 166 transcriptome datasets retrieved from the PLEXDB (Guo et al., 2016). They identified 968 candidates of regulators and represented a subnetwork for a regulatory gene FAC1 by superimposing information from its proteinprotein interaction (PPI) network and the differentially expressed genes of its mutant in F. graminearum. GENIE3 ${ }^{6}$, a treebased ML algorithm (Huynh-Thu et al., 2010), has been widely employed in recent GRN inference studies with both static and dynamic transcriptome data from various species (Banf and Rhee, 2017; Desai et al., 2017; Redekar et al., 2017). Huang et al. (2018) applied GENIE3 to infer GRNs with over 1,000 publicly available RNA-Seq data from various tissues such as leaf, root, shoot, apical meristem, and seed, and created four tissue-specific GRNs. They validated the predicted regulatory networks for transcription factors KN1 (KNOTTED1), FEA4 (fasciated ear4), and O2 (Opaque2), by using publicly available ChIP-seq datasets. Varala et al. (2018) applied dynamic factor graph (DFG) models (Mirowski and LeCun, 2009) to a finescale time-series transcriptome in response to nitrogen supply in Arabidopsis shoots and roots, and illustrated a GRN composed of nitrogen responsive TF and non-TF genes (Varala et al., 2018). They validated the predicted regulatory networks for transcription factors CRF4, SNZ, and CDF1, which showed early $\mathrm{N}$-response in shoots and roots, by using the TARGET (Transient Assay Reporting Genome-wide Effects of Transcription factors) method, and demonstrated that five key genes involved in $\mathrm{N}$ uptake and assimilation were included in the predicted and validated targets of these three TFs (Bargmann et al., 2013). These examples suggested that ML-based approaches provide opportunities to reconstruct GRNs from various types of transcriptome datasets, thereby assisting the identification of key

\footnotetext{
${ }^{5}$ http://www.cs.huji.ac.il/labs/compbio/minreg/

${ }^{6}$ https://bioconductor.org/packages/release/bioc/html/GENIE3.html
} 
TABLE 2 | Examples of combined approaches for GRN inference in plants and other species.

\begin{tabular}{|c|c|c|c|}
\hline Data type & Algorithm & Organisms & Reference \\
\hline \multirow[t]{2}{*}{ Time series } & Inferelator, TIGRESS, and GENIE3 & Arabidopsis thaliana & Foo et al., 2018 \\
\hline & ARACNE, GENIE3, TIGRESS, Partial correlation, and CLR & Glycine max & Redekar et al., 2017 \\
\hline \multirow[t]{2}{*}{ Time series (development) } & GRACE (Random forest and ensembles of Markov Random Fields) & Arabidopsis thaliana & Banf and Rhee, 2017 \\
\hline & & Drosophila melanogaster & \\
\hline
\end{tabular}

TF-genes involved in cellular systems related to various biological functions in plants.

\section{Combined Approaches}

Combinatorial use of multiple algorithms could be a promising strategy for GRN inference (Marbach et al., 2012; Table 2). Foo et al. (2018) employed three different algorithms, Inferelator, TIGRESS (Trustful Inference of Gene REgulation with Stability Selection $^{7}$ ) (Haury et al., 2012), and GENIE3, to infer GRNs involved in defense response of Arabidopsis with its microarraybased time-series transcriptome data (Foo et al., 2018), and verified a particular subnetwork using $\mathrm{Y} 1 \mathrm{H}$ assay, information from an Arabidopsis cistrome map, and gene expression profiles from overexpressors of a related gene. Redekar et al. (2017) used five different algorithms, ARACNE $^{8}$ (Margolin et al., 2006), GENIE3 (Huynh-Thu et al., 2010), TIGRESS (Haury et al., 2012), partial correlation (GeneTS ${ }^{9}$ ) (Schafer and Strimmer, 2005), and CLR (Faith et al., 2007), to infer the GRNs between TFs and co-expressed modules for seed development in soybean ${ }^{10}$, based on 60 RNA-Seq datasets (three biological replicates, five stages of developing seeds, and four experimental lines), and evaluated the resultant GRNs by comparative analysis with published GRNs of Arabidopsis (Redekar et al., 2017) ${ }^{10}$. Banf and Rhee developed a novel GRN inference strategy called GRACE (Gene Regulatory network inference ACcuracy Enhancement ${ }^{11}$ ), which generates GRNs through multiple steps to integrate various knowledge related to the regulation of gene expression: initial network prediction from gene expression data using a random forest regression model and integrating information related to gene regulation, subsequent network module extraction by meta-network construction based on information of functionally related genes, and further selection of regulatory links using ensembles of Markov Random Fields (Banf and Rhee, 2017). To infer the developmental GRN in Arabidopsis, the authors incorporated conserved sequence information in its promoter regions and experimentally determined cis-motifs for TFs, together with gene expression data from 83 tissues and stages, and obtained an initial GRN containing 325 regulators, 4,305 targets, and 10,098 links. To enhance confidence of the initially predicted GRN, the authors integrated knowledge from various information resources such as AraNet $^{12}$, ATRM (Arabidopsis

\footnotetext{
${ }^{7}$ http://projets.cbio.mines-paristech.fr/ ahaury/svn/dream5/html/index.html

${ }^{8} \mathrm{http}: / /$ califano.c2b2.columbia.edu/aracne/

${ }^{9} \mathrm{https} / / /$ bioconductor.org/packages/2.1/bioc/html/GeneTS.html

${ }^{10} \mathrm{https} / / /$ lilabatvt.github.io/LPANetwork/

${ }^{11}$ https://github.com/mbanf/GRACE

${ }^{12} \mathrm{http}: / / \mathrm{www}$. functionalnet.org/aranet/about.html
}

Transcriptional Regulatory Map ${ }^{13}$ ), $\mathrm{SUBA}^{14}$, and $\mathrm{AraCyc}{ }^{15}$, and demonstrated its potential to produce high-confidence regulatory networks, thereby suggesting a benefit of integration of multiple clues from various information resources to improve accuracy of the GRNs.

\section{GRNs WITH EMERGING APPLICATIONS}

In terms of recent advances in both resolution and throughput to acquire genome and transcriptome datasets (Reuter et al., 2015), and computational methodologies to analyze the datasets, GRNs have yielded various applications which allow us deeper understanding of cellular systems at population, life-course and single-cell level. Here, we highlight emerging applications of these approaches, through GRN reconstruction, from these three specific aspects.

\section{Population Transcriptomics for GRN Construction}

Population-scale transcriptome sequencing enables us to shed light on molecular consequences of regulatory variations in complex traits. Through transcriptome sequencing across mapping populations, eQTL analysis has been widely used to identify cis- and trans-QTLs, and reconstruct regulatory networks to mine genetic factors that determine various traits, including agronomic traits of crop species (Albert et al., 2018; Galpaz et al., 2018; Wang et al., 2018; Zhang et al., 2018). Moreover, a transcriptome-wide association study (TWAS) was proposed to identify associations between gene expression and traits (Gusev et al., 2016), and has recently been applied to construct GRNs. For example, integrating genome and transcriptome data of whole blood RNA-Seq samples across 3,072 unrelated individuals, Luijk et al. (2018) constructed a GRN that suggests 49 regulatory genes that affect transcriptional changes of their downstream genes. Moreover, population-scale transcriptome sequencing across multiple tissue types, have been applied to reconstruct GRNs through integration with other resources on molecular networks, such as PPI and TF motifs, to reveal tissue-specific gene regulation (Sonawane et al., 2017).

\footnotetext{
${ }^{13} \mathrm{http}: / /$ atrm.cbi.pku.edu.cn/

${ }^{14} \mathrm{http}: / /$ suba.live/

${ }^{15} \mathrm{https} / / /$ www.plantcyc.org/databases/aracyc/15.0
} 


\section{Spatial-Temporal GRNs at Single-Cell Level}

High-throughput sequencing applications at single-cell level have rapidly emerged, and enabled us to decipher GRNs underlying cellular heterogeneity (Liu and Trapnell, 2016; Libault et al., 2017; Dasgupta et al., 2018; Fiers et al., 2018). For GRN inference from single-cell transcriptome datasets, several computational algorithms have recently been developed. Chan et al. (2017) developed an algorithm, PIDC, which identifies regulatory relations between genes based on partial information decomposition (PID), and is applied to infer GRNs from single cell-qPCR datasets. SCENIC ${ }^{16}$ constructs GRNs and identifies cell-status based on scRNA-Seq data, which uses GENIE3 to predict TF targets based on co-expression, RcisTarget to assess TF-motif enrichment, and AUCell to assess regulon activities in each cell; it was recently applied to GRN analysis in a single-cell transcriptome from adult fly brain sampled across its lifespan (Davie et al., 2018). Although, till date, there are only a small number of scRNA-Seq datasets from higher plant species (Perroud et al., 2018), single-cell level highthroughput data in plants, and GRNs based on such datasets, will provide invaluable resource to facilitate in-depth elucidation of various cellular systems in plants (Efroni and Birnbaum, 2016).

\section{GRNs Throughout Life-Course}

Longitudinal transcriptome study provides insights into the trajectory of GRNs, underlying the biological phenomena throughout life-span/life-course, such as aging and phenology. Through a longitudinal transcriptome analysis of short-lived killifish, Nothobranchius furzeri, Baumgart et al. (2016) identified mitochondrial respiratory chain complex I genes as the hub in a co-expressed gene expression module that negatively correlated with its lifespan. For crop improvements, trajectories of physiological states, resulting from interaction between genetic and environmental factors, often influence the phenotypes of eventual agronomic traits; longitudinal study of cellular networks provides clues to identify gene-environment interactions associated with the phenotypic changes in crops (Mochida et al., 2015; Sun and Dinneny, 2018). Through construction of an integrated atlas of gene expression and regulatory networks in developing maize, Walley et al. (2016) demonstrated that integration of transcriptome, proteome, and phospho-proteome data can improve GRN inference. In tropical rice, as introduced in the previous sections, integrating time-series datasets of transcriptome, nucleosome-free chromatin from ATAC-seq, and known cis-motifs for TFs from five tropical rice cultivars under controlled and agricultural field conditions, Wilkins et al. (2016) constructed GRNs that represent relationships between the timing and gene expression in response to environmental changes. These examples from staple crops illuminate that combinatorial use of multiple omics data is a promising approach to improve the performance of GRN inference, as well as to mine better clues to improve agronomically important traits of crops under field conditions.

\footnotetext{
${ }^{16}$ https://github.com/aertslab/SCENIC
}

\section{CONCLUSION AND PERSPECTIVES}

In the last few years, approaches to reconstruct GRNs have advanced by synergistic innovation of high-throughput sequencing and computational techniques; GRNs have played crucial roles to elucidate cellular systems and identify key genes that manipulate cellular functions. A lot of statisticaland ML-based approaches have been proposed and applied to infer GRNs based on transcriptome datasets; these have contributed to identify regulatory relationships of genes involved in various biological phenomena in plants. Coupled with applications recently developed in high-throughput sequencing, GRNs dramatically improve their resolution with emerging aspects of transcriptomics, such as across accessions/individuals, cell types, and life stages, each of which provides opportunities to address challenges for these emerging areas in plant science.

Integration of GRNs and other networks, such as epigenetic, PPI, and metabolic networks, provides clues to identify molecular relations that function as interfaces, and will provide new insights into trans-omics networks across multiple omics layers (Yugi et al., 2016). ML has provided algorithms to find useful patterns from large and heterogeneous (unstructured) data, acquired through multiple high-throughput techniques (Ma et al., 2014; May, 2014; McCue and McCoy, 2017). Recently, ML-based approaches have been applied to extract features associated with cellular states and responses from high-throughput data, including transcriptomic and epigenomic data, and develop computational models that classify the cellular states and responses in applications such as precision oncology and drug development (Aliper et al., 2016; Malta et al., 2018). In plant science, ML-based integrative analysis of large-scale data from multiple omics spectra, such as genomic variations and molecular networks, as well as high-throughput phenomics, will enable us to decipher complex cellular systems and figure out molecular features associated with quantitative traits in plants and crops, and apply the results to design traits through optimizing GRNs in crop breeding. From the perspective of ML in GRN study, it will offer us algorithms not only for GRN inference but also for feature extraction across multi-dimensional datasets from various high-throughput experimental techniques.

\section{AUTHOR CONTRIBUTIONS}

$\mathrm{KM}, \mathrm{SK}, \mathrm{KI}$, and RN conceived the study, performed the research, and wrote the manuscript.

\section{FUNDING}

The work was partially supported by Grant-in-Aid for Scientific Research (B) (Grant No. 15KT0038 to KM) of the Japan Society for the Promotion of Science (JSPS), and by the Advanced Low Carbon Technology Research and Development Program (ALCA, J2013403C to KM and RN) of the Japan Science and Technology Agency (JST). This work was also supported by CREST, JST. 


\section{REFERENCES}

Albert, E., Duboscq, R., Latreille, M., Santoni, S., Beukers, M., Bouchet, J. P., et al. (2018). Allele specific expression and genetic determinants of transcriptomic variations in response to mild water deficit in tomato. Plant J. 96, 635-650. doi: 10.1111/tpj.14057

Aliper, A., Plis, S., Artemov, A., Ulloa, A., Mamoshina, P., and Zhavoronkov, A. (2016). Deep learning applications for predicting pharmacological properties of drugs and drug repurposing using transcriptomic data. Mol. Pharm. 13, 2524-2530. doi: 10.1021/acs.molpharmaceut.6b00248

Banf, M., and Rhee, S. Y. (2017). Enhancing gene regulatory network inference through data integration with markov random fields. Sci. Rep. 7:41174. doi: $10.1038 /$ srep 41174

Barabasi, A. L., and Oltvai, Z. N. (2004). Network biology: understanding the cell's functional organization. Nat. Rev. Genet. 5, 101-113. doi: 10.1038/nrg1272

Bargmann, B. O., Marshall-Colon, A., Efroni, I., Ruffel, S., Birnbaum, K. D., Coruzzi, G. M., et al. (2013). TARGET: a transient transformation system for genome-wide transcription factor target discovery. Mol. Plant 6, 978-980. doi: $10.1093 / \mathrm{mp} / \mathrm{sst} 010$

Basnet, R. K., Del Carpio, D. P., Xiao, D., Bucher, J., Jin, M., Boyle, K., et al. (2016). A systems genetics approach identifies gene regulatory networks associated with fatty acid composition in brassica rapa seed. Plant Physiol. 170, 568-585. doi: 10.1104/pp.15.00853

Baumgart, M., Priebe, S., Groth, M., Hartmann, N., Menzel, U., Pandolfini, L., et al. (2016). Longitudinal RNA-seq analysis of vertebrate aging identifies mitochondrial complex i as a small-molecule-sensitive modifier of lifespan. Cell Syst. 2, 122-132. doi: 10.1016/j.cels.2016.01.014

Blais, A., and Dynlacht, B. D. (2005). Constructing transcriptional regulatory networks. Genes Dev. 19, 1499-1511. doi: 10.1101/gad.1325605

Blum, C. F., Heramvand, N., Khonsari, A. S., and Kollmann, M. (2018). Experimental noise cutoff boosts inferability of transcriptional networks in large-scale gene-deletion studies. Nat. Commun. 9:133. doi: 10.1038/s41467017-02489-x

Calabrese, G. M., Mesner, L. D., Stains, J. P., Tommasini, S. M., Horowitz, M. C., Rosen, C. J., et al. (2017). Integrating gwas and co-expression network data identifies bone mineral density genes SPTBN1 and MARK3 and an osteoblast functional module. Cell Syst. 4:46-59.e4. doi: 10.1016/j.cels.2016. 10.014

Camacho, D. M., Collins, K. M., Powers, R. K., Costello, J. C., and Collins, J. J. (2018). Next-Generation machine learning for biological networks. Cell 173, 1581-1592. doi: 10.1016/j.cell.2018.05.015

Chan, T. E., Stumpf, M. P. H., and Babtie, A. C. (2017). Gene regulatory network inference from single-cell data using multivariate information measures. Cell Syst. 5, 251-267.e3. doi: 10.1016/j.cels.2017.08.014

Dasgupta, S., Bader, G. D., and Goyal, S. (2018). Single-cell RNA sequencing: a new window into cell scale dynamics. Biophys. J. 115, 429-435. doi: 10.1016/j.bpj. 2018.07.003

Davie, K., Janssens, J., Koldere, D., De Waegeneer, M., Pech, U., Kreft, L., et al. (2018). A single-cell transcriptome atlas of the aging drosophila brain. Cell 174:982-998.e20. doi: 10.1016/j.cell.2018.05.057

de Luis Balaguer, M. A., Fisher, A. P., Clark, N. M., Fernandez-Espinosa, M. G., Moller, B. K., Weijers, D., et al. (2017). Predicting gene regulatory networks by combining spatial and temporal gene expression data in Arabidopsis root stem cells. Proc. Natl. Acad. Sci. U.S.A. 114, E7632-E7640. doi: 10.1073/pnas. 1707566114

Desai, J. S., Sartor, R. C., Lawas, L. M., Jagadish, S. V. K., and Doherty, C. J. (2017). Improving gene regulatory network inference by incorporating rates of transcriptional changes. Sci. Rep. 7:17244. doi: 10.1038/s41598-017-17 143-1

Dewey, G. T., and Galas, D. J. (2010). "Gene Regulatory Networks," in Madame Curie Bioscience Database (Austin, TX: Landes Bioscience). Available at: https://www.ncbi.nlm.nih.gov/books/NBK5974/

Efroni, I., and Birnbaum, K. D. (2016). The potential of single-cell profiling in plants. Genome Biol. 17:65. doi: 10.1186/s13059-016-0931-2

Faith, J. J., Hayete, B., Thaden, J. T., Mogno, I., Wierzbowski, J., Cottarel, G., et al. (2007). Large-scale mapping and validation of Escherichia coli transcriptional regulation from a compendium of expression profiles. PLoS Biol. 5:e8. doi: 10.1371/journal.pbio.0050008
Fiers, M., Minnoye, L., Aibar, S., Bravo Gonzalez-Blas, C., Kalender Atak, Z., and Aerts, S. (2018). Mapping gene regulatory networks from single-cell omics data. Brief Funct. Genomics 17, 246-254. doi: 10.1093/bfgp/elx046

Foo, M., Gherman, I., Zhang, P., Bates, D. G., and Denby, K. J. (2018). A framework for engineering stress resilient plants using genetic feedback control and regulatory network rewiring. ACS Synth. Biol. 7, 1553-1564. doi: 10.1021/ acssynbio.8b00037

Fuxman Bass, J. I., Sahni, N., Shrestha, S., Garcia-Gonzalez, A., Mori, A., Bhat, N., et al. (2015). Human gene-centered transcription factor networks for enhancers and disease variants. Cell 161, 661-673. doi: 10.1016/j.cell.2015.03.003

Galpaz, N., Gonda, I., Shem-Tov, D., Barad, O., Tzuri, G., Lev, S., et al. (2018). Deciphering genetic factors that determine melon fruit-quality traits using RNA-Seq-based high-resolution QTL and eQTL mapping. Plant J. 94, 169-191. doi: $10.1111 /$ tpj. 13838

Greenfield, A., Hafemeister, C., and Bonneau, R. (2013). Robust data-driven incorporation of prior knowledge into the inference of dynamic regulatory networks. Bioinformatics 29, 1060-1067. doi: 10.1093/bioinformatics/ btt099

Guo, L., Zhao, G., Xu, J. R., Kistler, H. C., Gao, L., and Ma, L. J. (2016). Compartmentalized gene regulatory network of the pathogenic fungus Fusarium graminearum. New Phytol. 211, 527-541. doi: 10.1111/nph.13912

Gusev, A., Ko, A., Shi, H., Bhatia, G., Chung, W., Penninx, B. W., et al. (2016). Integrative approaches for large-scale transcriptome-wide association studies. Nat. Genet. 48, 245-252. doi: 10.1038/ng.3506

Hanson, C., Cairns, J., Wang, L., and Sinha, S. (2018). Principled multi-omic analysis reveals gene regulatory mechanisms of phenotype variation. Genome Res. 28, 1207-1216. doi: 10.1101/gr.227066.117

Haury, A. C., Mordelet, F., Vera-Licona, P., and Vert, J. P. (2012). TIGRESS: trustful inference of gene regulation using stability selection. BMC Syst. Biol. 6:145. doi: $10.1186 / 1752-0509-6-145$

Hickman, R., Van Verk, M. C., Van Dijken, A. J. H., Mendes, M. P., Vroegop-Vos, I. A., Caarls, L., et al. (2017). Architecture and dynamics of the jasmonic acid gene regulatory network. Plant Cell 29, 2086-2105. doi: 10.1105/tpc.16.00958

Huang, J., Zheng, J., Yuan, H., and McGinnis, K. (2018). Distinct tissue-specific transcriptional regulation revealed by gene regulatory networks in maize. $B M C$ Plant Biol. 18:111. doi: 10.1186/s12870-018-1329-y

Huynh-Thu, V. A., Irrthum, A., Wehenkel, L., and Geurts, P. (2010). Inferring regulatory networks from expression data using tree-based methods. PLoS One 5:e12776. doi: 10.1371/journal.pone.0012776

Ikeuchi, M., Shibata, M., Rymen, B., Iwase, A., Bagman, A. M., Watt, L., et al. (2018). A gene regulatory network for cellular reprogramming in plant regeneration. Plant Cell Physiol. 59, 765-777. doi: 10.1093/pcp/pcy013

Kemmeren, P., Sameith, K., van de Pasch, L. A., Benschop, J. J., Lenstra, T. L., Margaritis, T., et al. (2014). Large-scale genetic perturbations reveal regulatory networks and an abundance of gene-specific repressors. Cell 157, 740-752. doi: 10.1016/j.cell.2014.02.054

Koda, S., Onda, Y., Matsui, H., Takahagi, K., Yamaguchi-Uehara, Y., Shimizu, M., et al. (2017). Diurnal transcriptome and gene network represented through sparse modeling in brachypodium distachyon. Front. Plant Sci. 8:2055. doi: 10.3389/fpls.2017.02055

Libault, M., Pingault, L., Zogli, P., and Schiefelbein, J. (2017). Plant systems biology at the single-cell level. Trends Plant Sci. 22, 949-960. doi: 10.1016/j.tplants.2017. 08.006

Liu, F., Zhang, S. W., Guo, W. F., Wei, Z. G., and Chen, L. (2016). Inference of gene regulatory network based on local bayesian networks. PLoS Comput. Biol. 12:e1005024. doi: 10.1371/journal.pcbi.1005024

Liu, S., and Trapnell, C. (2016). Single-cell transcriptome sequencing: recent advances and remaining challenges. F1000Res 5:F1000FacultyRev-182. doi: 10. 12688/f1000research.7223.1

Lopez-Maury, L., Marguerat, S., and Bahler, J. (2008). Tuning gene expression to changing environments: from rapid responses to evolutionary adaptation. Nat. Rev. Genet. 9, 583-593. doi: 10.1038/nrg2398

Luijk, R., Dekkers, K. F., van Iterson, M., Arindrarto, W., Claringbould, A., Hop, P., et al. (2018). Genome-wide identification of directed gene networks using largescale population genomics data. Nat. Commun. 9:3097. doi: 10.1038/s41467018-05452-6

Ma, C., Zhang, H. H., and Wang, X. (2014). Machine learning for big data analytics in plants. Trends Plant Sci. 19, 798-808. doi: 10.1016/j.tplants.2014.08.004 
Malta, T. M., Sokolov, A., Gentles, A. J., Burzykowski, T., Poisson, L., Weinstein, J. N., et al. (2018). Machine learning identifies stemness features associated with oncogenic dedifferentiation. Cell 173, 338-354.e15. doi: 10.1016/j.cell.2018.03. 034

Marbach, D., Costello, J. C., Kuffner, R., Vega, N. M., Prill, R. J., Camacho, D. M., et al. (2012). Wisdom of crowds for robust gene network inference. Nat. Methods 9, 796-804. doi: 10.1038/nmeth.2016

Marchand, G., Huynh-Thu, V. A., Kane, N. C., Arribat, S., Vares, D., Rengel, D., et al. (2014). Bridging physiological and evolutionary time-scales in a gene regulatory network. New Phytol. 203, 685-696. doi: 10.1111/nph. 12818

Margolin, A. A., Nemenman, I., Basso, K., Wiggins, C., Stolovitzky, G., Dalla Favera, R., et al. (2006). ARACNE: an algorithm for the reconstruction of gene regulatory networks in a mammalian cellular context. BMC Bioinformatics 7(Suppl. 1):S7. doi: 10.1186/1471-2105-7-S1-S7

May, M. (2014). Big biological impacts from big data. Science 344, 1298-1301. doi: 10.1126/science.opms.p1400086

McCue, M. E., and McCoy, A. M. (2017). The scope of big data in one medicine: unprecedented opportunities and challenges. Front. Vet. Sci. 4:194. doi: 10. 3389/fvets.2017.00194

Mirowski, P., and LeCun, Y. (2009). (Dynamic) Factor Graphs for Time Series Modeling. Heidelberg: Springer, 128-143. doi: 10.1007/978-3-642-04174-7_9

Mochida, K., Saisho, D., and Hirayama, T. (2015). Crop improvement using life cycle datasets acquired under field conditions. Front. Plant Sci. 6:740. doi: 10.3389/fpls.2015.00740

Ni, Y., Aghamirzaie, D., Elmarakeby, H., Collakova, E., Li, S., Grene, R., et al. (2016). A machine learning approach to predict gene regulatory networks in seed development in Arabidopsis. Front. Plant Sci. 7:1936. doi: 10.3389/fpls. 2016.01936

Omranian, N., Eloundou-Mbebi, J. M., Mueller-Roeber, B., and Nikoloski, Z. (2016). Gene regulatory network inference using fused LASSO on multiple data sets. Sci. Rep. 6:20533. doi: 10.1038/srep20533

Perroud, P. F., Haas, F. B., Hiss, M., Ullrich, K. K., Alboresi, A., Amirebrahimi, M., et al. (2018). The Physcomitrella patens gene atlas project: large-scale RNA-seq based expression data. Plant J. 95, 168-182. doi: 10.1111/tpj. 13940

Redekar, N., Pilot, G., Raboy, V., Li, S., and Saghai Maroof, M. A. (2017). Inference of transcription regulatory network in low phytic acid soybean seeds. Front. Plant Sci. 8:2029. doi: 10.3389/fpls.2017.02029

Reuter, J. A., Spacek, D. V., and Snyder, M. P. (2015). High-throughput sequencing technologies. Mol. Cell. 58, 586-597. doi: 10.1016/j.molcel.2015.05.004

Schafer, J., and Strimmer, K. (2005). A shrinkage approach to large-scale covariance matrix estimation and implications for functional genomics. Stat. Appl. Genet. Mol. Biol. 4:Article32. doi: 10.2202/1544-6115.1175

Sonawane, A. R., Platig, J., Fagny, M., Chen, C. Y., Paulson, J. N., Lopes-Ramos, C. M., et al. (2017). Understanding tissue-specific gene regulation. Cell Rep. 21, 1077-1088. doi: 10.1016/j.celrep.2017.10.001
Sun, Y., and Dinneny, J. R. (2018). Q\&A: how do gene regulatory networks control environmental responses in plants? BMC Biol. 16:38. doi: 10.1186/s12915-0180506-7

van Dam, S., Vosa, U., van der Graaf, A., Franke, L., and de Magalhaes, J. P. (2018). Gene co-expression analysis for functional classification and genedisease predictions. Brief. Bioinform. 19, 575-592. doi: 10.1093/bib/bbw139

Varala, K., Marshall-Colon, A., Cirrone, J., Brooks, M. D., Pasquino, A. V., Leran, S., et al. (2018). Temporal transcriptional logic of dynamic regulatory networks underlying nitrogen signaling and use in plants. Proc. Natl. Acad. Sci. U.S.A. 115, 6494-6499. doi: 10.1073/pnas.1721487115

Walley, J. W., Sartor, R. C., Shen, Z., Schmitz, R. J., Wu, K. J., Urich, M. A., et al. (2016). Integration of omic networks in a developmental atlas of maize. Science 353, 814-818. doi: 10.1126/science.aag1125

Wang, X., Chen, Q., Wu, Y., Lemmon, Z. H., Xu, G., Huang, C., et al. (2018). Genome-wide analysis of transcriptional variability in a large maize-teosinte population. Mol. Plant 11, 443-459. doi: 10.1016/j.molp.2017.12.011

Webb, S. (2018). Deep learning for biology. Nature 554, 555-557. doi: 10.1038/ d41586-018-02174-z

Wilkins, O., Hafemeister, C., Plessis, A., Holloway-Phillips, M. M., Pham, G. M., Nicotra, A. B., et al. (2016). EGRINs (Environmental Gene Regulatory Influence Networks) in rice that function in the response to water deficit, high temperature, and agricultural environments. Plant Cell 28, 2365-2384. doi: $10.1105 /$ tpc. 16.00158

Xiong, W., Wang, C., Zhang, X., Yang, Q., Shao, R., Lai, J., et al. (2017). Highly interwoven communities of a gene regulatory network unveil topologically important genes for maize seed development. Plant J. 92, 1143-1156. doi: $10.1111 /$ tpj. 13750

Yugi, K., Kubota, H., Hatano, A., and Kuroda, S. (2016). Trans-omics: how to reconstruct biochemical networks across multiple 'Omic'. Layers. Trends Biotechnol. 34, 276-290. doi: 10.1016/j.tibtech.2015.12.013

Zhang, J., Yang, Y., Zheng, K., Xie, M., Feng, K., Jawdy, S. S., et al. (2018). Genomewide association studies and expression-based quantitative trait loci analyses reveal roles of HCT2 in caffeoylquinic acid biosynthesis and its regulation by defense-responsive transcription factors in Populus. New Phytol 220, 502-516. doi: $10.1111 /$ nph.15297

Conflict of Interest Statement: The authors declare that the research was conducted in the absence of any commercial or financial relationships that could be construed as a potential conflict of interest.

Copyright (c) 2018 Mochida, Koda, Inoue and Nishii. This is an open-access article distributed under the terms of the Creative Commons Attribution License (CC BY). The use, distribution or reproduction in other forums is permitted, provided the original author(s) and the copyright owner(s) are credited and that the original publication in this journal is cited, in accordance with accepted academic practice. No use, distribution or reproduction is permitted which does not comply with these terms. 